\title{
L'emofiltrazione come test diagnostico dell'iperparatiroidismo secondario
}

\author{
V. Panichi', A.M. Bianchi ${ }^{1}$, B. Andreini ${ }^{1}$, M. Migliori ${ }^{1}$, S. De Pietro ${ }^{1}$, L. Casarosa ${ }^{1}$, \\ L. Giovannini' ${ }^{2}$ R. Palla ${ }^{1}$
}

${ }^{1}$ Istituto Clinica Medica II, ${ }^{2}$ Istituto di Farmacologia - Università di Pisa

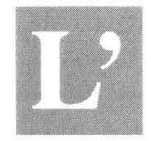

iperparatiroidismo secondario è una frequente complicanza nel paziente uremico e la riduzione del calcio ionizzato plasmatico ne rappresenta il principale evento patogenetico (1). Numerosi altri fattori sono stati chiamati in causa fra i quali l'accumulo del fosfato, un alterato metabolismo della vitamina $\mathrm{D}$, la resistenza scheletrica al paratormone ed una ridotta degradazione ormonale secondaria all'insufficienza renale (2). Queste alterazioni del metabolismo fosfo-calcico hanno negli anni stimolato la ricerca di nuove formulazioni per i bagni dialisi e di diverse strategie dialitiche al fine di ottenere un bilancio del calcio positivo associato ad un elevata rimozione intradialitica del fosfato (36). Nonostante il vasto uso dell'emofiltrazione nella pratica emodialitica sono disponibili poche informazioni sulle modificazioni indotte da questa tecnica sui livelli plasmatici di calcio-fosforo e paratormone $(8,9)$. In questo studio abbiamo valutato durante una seduta di emofiltrazione la cinetica del calcio ionizzato e del fosfato, l'effetto inibitorio dell'aumento del calcio ionizzato sull'increzione del PTH intatto e la sua rimozione convettiva. Abbiamo inoltre esaminato le variazioni intradialitiche di altre molecole calciotrope quali osteocalcina, procollageno I e III, fosfatasi alcalina e 1-25 vitamina $\mathrm{D}$.

\section{Materiali e metodi}

\section{Pazienti}

31 pazienti in trattamento emodialitico cronico da almeno sei mesi sono stati studiati durante una singola seduta di emofiltrazione intrasettimanale; la loro età media era 54 anni con un range tra 25 e 68 anni. Tutti i farmaci in grado di interferire con il metabolismo calcio fosforo (in particolare vitamina $\mathrm{D}$ e chelanti del fosfato) erano sospesi almeno un mese prima delllo studio.

\section{Tecnica dialitica}

È stata eseguita emofiltrazione in postdiluizione con un emofiltro in poliamide di $1.9 \mathrm{~m}$ (Gambro FH 88), con flusso sangue di $450 \pm 50 \mathrm{ml} / \mathrm{min}$ e flusso del reinfusato di $120 \pm 20 \mathrm{ml} / \mathrm{min}$. La composizione del liquido di reinfusione è mostrata nella Tabella I.

\section{Prelievi}

All'inizio, dopo un'ora ed alla fine della dialisi sono stati prelevati campioni per il calcio totale, calcio ionizzato, fosfato, fosfatasi alcalina, PTH molecola intatta

\section{TABELLA I - COMPOSIZIONE DEL LIQUIDO DI REINFU- SIONE IN HF}

\begin{tabular}{lr}
\hline Lattato $(\mathrm{mmol} / \mathrm{L})$ & 42 \\
Sodio $(\mathrm{mEq} / \mathrm{L})$ & 140 \\
Potassio $(\mathrm{mEq} / \mathrm{L})$ & 1.05 \\
Calcio $(\mathrm{mEq} / \mathrm{L})$ & 4.50 \\
Cloro $(\mathrm{mEq} / \mathrm{L})$ & 105 \\
Magnesio $(\mathrm{mEq} / \mathrm{L})$ & 1.00 \\
Glucosio $(\mathrm{g} / \mathrm{L})$ & 2.00
\end{tabular}

(PTHi), procollageno I e III, osteocalcina (GLA) e 1-25 vitamina D. Dopo un'ora ed al termine della dialisi sono state determinate nell'ultrafiltrato le concentrazioni di calcio ionizzato e PTHi; il calcio ionizzato è stato inoltre dosato nel liquido di reinfusione.

\section{Metodi di laboratorio}

Il calcio totale e ionizato sono stati determinati con un calcio ionometro (Nova 7 Pabish); in 40 controlli normali la media \pm 2 deviazioni standard nel nostro laboratorio per il calcio ione è risultata $1.26 \pm 0.05 \mathrm{mmol} / \mathrm{L}$. Il PTHi è stato determinato con un me- 
todo immunoradiometrico (10) (Allegro, Nichols), i valori normali del nostro laboratorio erano compresi tra 10-65 pg/ml, con un limite di detezione di $5 \mathrm{pg} / \mathrm{ml}$. Il procollageno I e III (11), l'osteocalcina e $1-25$ vitamina D sono stati determinati con radioimmunoassay (RIA); il fosfato e la fosfatasi alcalina con le comuni metodiche di laboratorio usando un Hitachi Autoanalyzer.

\section{Statistica}

I risultati sono stati espressi come media \pm deviazione standard. L'analisi statistica è stata eseguita utilizzando il test di Student per dati appaiati. L'inibizione massimale del PTHi è stata definita come la percentuale del massimo decremento del PTHi dai valori basali.

\section{Risultati}

Nella Tabella II sono mostrati i dati relativi alle variazioni del calcio totale e io- nizzato e del PTHi. In base ai valori iniziali del PTHi abbiamo diviso i pazienti in due gruppi: il primo gruppo di 26 con un valore basale di PTHi di $123 \pm 111$ $\mathrm{pg} / \mathrm{ml}$ ed un calcio ionizzato di $1.17 \pm$ $0.08 \mathrm{mmol} / \mathrm{L}$ ed un secondo gruppo di 5 pazienti con PTHi basale di $1015 \pm 382$ $\mathrm{pg} / \mathrm{ml}$ ed un calcio ionizzato di $1.28 \pm$ $0.12 \mathrm{mmol} / \mathrm{L}$.

I pazienti del primo gruppo (Fig. 1b) hanno mostrato una risposta fisiologica delle paratiroidi con marcata riduzione del PTHi dopo un'ora ed inibizione massimale dell' $88 \%$. Nel secondo gruppo nonostante l'incremento del calcio ionizzato da $1.28 \pm 0.12$ a $1.46 \pm 0.08 \mathrm{mmol} / \mathrm{L}$ (Fig. 1a) il feed back fisiologico calcioparatormone era perso non evidenziandosi un significativo calo del PTHi.

La concentrazione del calcio ionizzato nel liquido di reinfusione è risultata $1.61 \pm$ $0.02 \mathrm{mmol} / \mathrm{L}$ con un bilancio di massa intradialitico del calcio ionizzato positivo di $8 \pm 2 \mathrm{mmol} / \mathrm{L}(44 \pm 8 \mathrm{mmol}$ infuse e $36 \pm$ $6 \mathrm{mmol}$ rimosse). La rimozione convettiva del PTHi è stata di $9 \pm 3 \mathrm{pg} / \mathrm{ml}$.

Nella Tabella III sono indicate le variazioni intradialitiche dell'osteocalcina

TABELLA II - VARIAZIONI INTRADIALITICHE DEL CALCIO IONIZZATO (iCa) E PTHi NEL GRUPPO 1 (RESPONDERS, N=26) E NEL GRUPPO 2 (NON RESPONDERS, N=5)

\begin{tabular}{lccc}
\hline & Basale & Prima ora & Fine dialisi \\
\hline Gruppo 1 & & & \\
iCa mmol/L & $1.17 \pm 0.08$ & $1.38 \pm 0.06^{*}$ & $1.42 \pm 0.07^{*}$ \\
PTHi pg/ml & $123.53 \pm 111$ & $39.53 \pm 34^{*}$ & $35.7 \pm 28^{*}$ \\
Gruppo 2 & & & \\
iCa mmol/L & $1.28 \pm 0.12$ & $1.36 \pm 0.11^{*}$ & $1.46 \pm 0.8^{*}$ \\
PTHi pg/ml & $1015 \pm 382$ & $523 \pm 227^{*}$ & $554 \pm 244^{*}$ \\
\hline
\end{tabular}

$* \mathrm{p}<0.05$

TABELLA III - VARIAZIONI INTRADIALITICHE DELL'OSTEOCALCINA (GLA), PROCOLLAGENO I E III, 1,25-DIIDROSSI VIT. D, FOSFATASI ALCALINA (ALP) E FOSFATO IN TUTTI I PAZIENTI

\begin{tabular}{lccc}
\hline & Basale & Prima ora & Fine dialisi \\
\hline GLA ng/ml & $11.25 \pm 8.21$ & $6.18 \pm 5.64^{*}$ & $3.82 \pm 4.0^{*}$ \\
Procollageno I mcg/L & $150 \pm 41$ & $130 \pm 38$ & $103 \pm 35^{*}$ \\
Procollageno III mcg/L & $9.76 \pm 2.25$ & $8.78 \pm 2.47$ & $8.35 \pm 2.42$ \\
1,25-diidrossi vit.D pg/ml & $10.86 \pm 3.3$ & $12.1 \pm 2.1$ & $13.6 \pm 3.9^{*}$ \\
ALP U/L & $237 \pm 130$ & $249 \pm 135$ & $252 \pm 159$ \\
Fosfato mEq/L & $2.54 \pm 1.19$ & $1.44 \pm 0.46^{*}$ & $1.27 \pm 0.35$ \\
\hline
\end{tabular}

$* \mathrm{p}<0.05$
(GLA), del procollageno I e III, della vitamina D, della fosfatasi alcalina e della fosfatemia.

\section{Conclusioni}

Una singola seduta di emofiltrazione è in grado di ridurre i livelli sierici del PTHi. Il decremento è in relazione alla rimozione convettiva dell'ormone ed alla soppressione biologica dell'attività paratiroidea da parte del calcio ione. Abbiamo utilizzato una singola seduta di emofiltrazione come test clinico per l'esplorazione funzionale delle paratiroidi che ci ha consentito di individuare due gruppi di pazienti affetti da iperparatiroidismo. I pazienti del primo gruppo hanno mostrato una risposta fisiologica della ghiandola sensibile al calcio-ione con inibizione massimale dell'increzione ormonale dell' $88 \%$; in questi pazienti la supplementazione con il calcio è un presidio terapeutico obbligatorio nel controllo dell'iperparatiroidismo. I pazienti del secondo gruppo hanno invece mostrato la perdita del feed-back fisiologico con valori di PTHi a fine dialisi dieci volte più alti del normale ed inibizione massimale non superiore al $45 \%$ nonostante un bilancio di massa del calcio positivo. In questi pazienti si è verificata l'autonomizzazione della funzione ghiandolare con perdita della sensibilità alle variazioni del calcio ione. ̇̀ importante nel tentativo di ridurre l'ipereattivita delle paratiroidi correggere il deficit di vitamina $\mathrm{D}$, dimostrato anche dai bassi livelli ematici di calcitriolo. Numerosi studi a lungo termine suggeriscono comunque la paratiroidectomia nei pazienti con grado molto severo di iperparatiroidismo, nei quali la supplementazione di vitamina $D$ può non essere sufficiente. Nell'insufficienza renale sono stati descritti $(12,13)$ elevati valori di osteocalcina e procollageno I e III sensibili marker di turn-over osseo (14), mentre livelli elevati di osteocalcina sono stati anche associati al rimodellamento osseo dell'iperparatiroidismo secondario (15). Nel nostro studio abbiamo osservato un calo importante dei livelli di osteocalcina (peso molecolare intorno a $5900 \mathrm{D}$ ) con una riduzione lieve e non statisticamente significativa di procollageno I e III ( peso molecolare intorno a 100.000 D). Queste variazioni sono interamente dovute alla tecnica dialitica ed al peso 


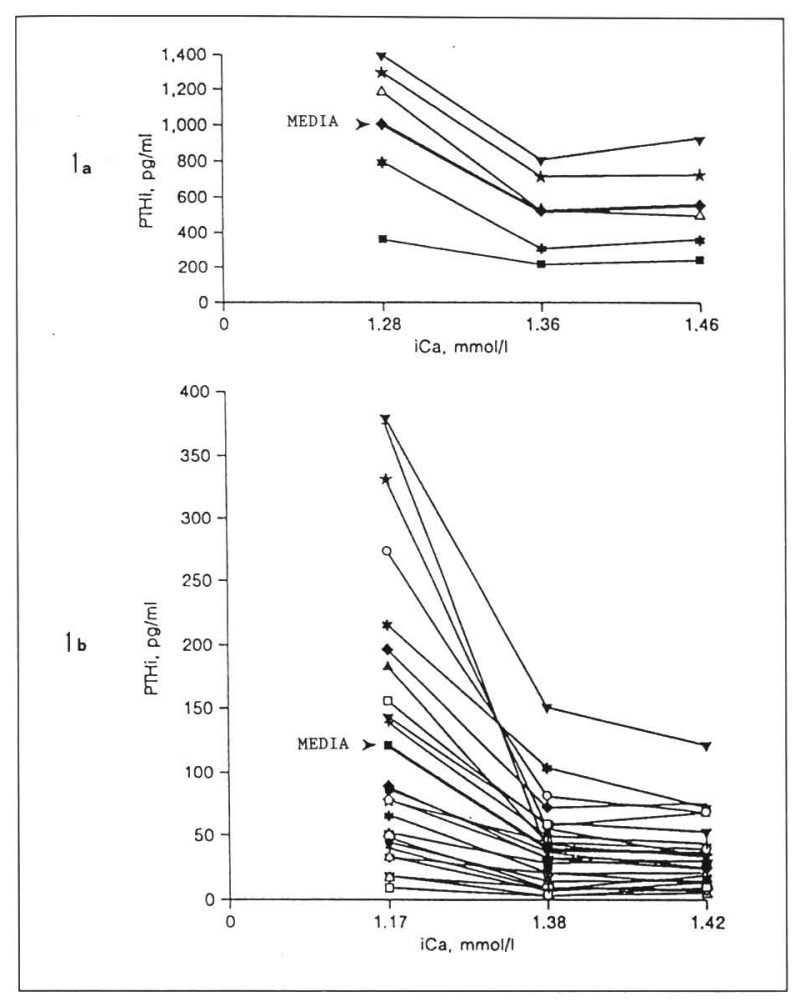

Fig. 1 - Risposta delle paratiroidi all incremento del calcio ionizzato. a: gruppo 1 (responders). $b$ : gruppo 2 (non responders).

molecolare confermando che queste molecole, certamente utili nelle condizioni caratterizzate da incremento della produzione di connettivo (16), non possono essere utilizzate come marker a breve termine di rimodellamento osseo nei pazienti dializzati.

In conclusione proponiamo l'uso di una singola seduta di emofiltrazione come test diagnostico della sensibilità paratiroidea al calcio.

\section{BIBLIOGRAFIA}

1.

Brown EM, Wilkson RE, Eastmann RC, Pallotta J, Marinick SP. Abnormal regulation of parathyroid hormone release by calcium in secondary hyperparathyroidism due to chronic renal failure. Clin Endocrinol Metab 1982; 54: 172-9.

2. Felsenfeld AJ, Llach F. Parathyroid gland function in chronic renal failure. Kidney Int 1993; 43: 771-89.

3. Goldsmith RS, Furzyfer J, Hohnson WJ, Beeler GW, Taylor
WF. Calcium flux during hemodialysis. Nephron 1978; 20: 132-40.

4. Carney SL, Jilles AHG. Effect of an optimum dialysis fluid calcium concentration on calcium mass transfer during maintenance hemodialysis. Clin Nephrol 1985; 24: $28-30$.

5. D'Amour P, Jobin J, Hamel L. L'Ecuyer N. iPTH values during hemodialysis. Role of ionized calcium, dialysis membranes and iPTH assays. Kidney Int 1990; 38: 308-14.

6. Argiles A, Kerr PG, Canaud B, Flavier JL, Mion C. Calcium kinetics and long-term effects of lowering dialysate calcium concentration. Kidney Int 1993; 43: 630-40.

7. Baldamus CA, Quellhorst E. Outcome of long-term hemofiltration. Kidney Int 1985; 28 (suppl 17): 41-6.

8. Matthaei D, Kramer P, Langescheid C, et al. Parathyroid hormone, calcium, phosphate balance in hemofiltration. J Dial 1977; 1: 64051.

9. Pierides AM, Giacherio D,
Schniepp B, Burrit M. Calcium, phosphourus and parathyroid hormone metabolism in chronic hemofiltration. In: Schaefer K ed. Hemofiltration. Sympon Hemofiltration, Berlin 1981. Contrib Nephrol. Basel: Karger, 1982; 32: 77-85.

10. Hackeng WHL, Lips P, Coen J, Lips CJM. Clinical implications of estimation of intact parathyroid hormone (PTH) versus total immunoreactive PTH in normal subjects and hyperparathyroidism patients. J Clin Endocrinol Metab 1986; 63: 447.

11. Melkko J, Nlemy S, Risteli L, Risteli J. Radioimmunoassay of the carboxy-terminal propeptide of human type I procollagen. Clin Chem 1990; 36: 1328-32.

12. Malluche HH, Faugere MC, Fanti P, Price PA. Plasma levels of bone GLA-protein reflect bone formation in patients on chronic maintenance hemodialysis. Kidney Int 1984; 26: 869-74.

13. Coen G, Mazzaferro S, Ballanti P, et al. Procollagen type I C-terminal extension peptide in predialysis chronic renal failure. Am J Nephrol 1992; 12: 246-51.

14. Charon SA, Delmas PD, Malaval L, et al. Serum bone GLA-protein in renal osteodistrophy: Comparison with bone histomorphometry. J Clin Endocrinol Metab 1986; 63: 892-7.

15. Martinez ME, Selgas R, Miguel JL, Balaguer G, Sanchez-Cabezudo MJ, Llach F. Osteocalcin levels in uremic patients: Influence of calcitriol treatment through different routes and type of dialysis. Nephron 1991; 59: 429-33.

16. Bolarin DM, Savolainem ER, Kivirikko KI. Enzymes of collagen biosinthesis and type III procollagen aminopropeptide in serum from $\mathrm{Ni}$ gerians hepatocellular carcinoma and others malignant diseases. Int J Cancer 1982; 29: 401-5. 